\title{
The Effect of Population Density on the Performance of a Spatial Social Network Algorithm for Multi-Objective Optimisation
}

\author{
Andrew Lewis
}

\begin{abstract}
Particle Swarm Optimisation (PSO) is increasingly being applied to optimisation of multi-objective problems in engineering design and scientific investigation. This paper investigates the behaviour of a novel algorithm based on an extension of the concepts of spatial social networks using a model of the behaviour of locusts and crickets. In particular, observation of locust swarms suggests a specific dependence on population density for ordered behaviour. Computational experiments demonstrate that both the new, spatial, social network algorithm and a conventional MOPSO algorithm exhibit improved performance with increased swarm size and crowding. This observation may have particular significance for design of some forms of distributed PSO algorithms.
\end{abstract}

\section{INTRODUCTION}

Particle Swarm Optimisation (PSO) is a relatively new class of optimisation algorithms, introduced by Kennedy and Eberhart in 1995 [8]. This technique is based upon the social interaction of members of a population or swarm, usually referred to as particles. Applied to optimisation, the swarm of particles travels through the problem parameter space, its motion being guided by its own, past performance and another selected guide particle, most often the particle in the swarm with the current best fitness. Widely successful for problems involving a single objective [11], [6], extension to multiobjective optimisation problems raises the problem of how to choose the guide particle, given that "fitness" is generally a compromise between different, often competing objectives. Several different methods of choosing guide particles have been investigated [10], [7] but it is believed no selection scheme will give adequate performance on all test problems.

Previously, the author has suggested a novel approach to this problem [9], based on an extension of the concepts of spatial social networks [4]. Using spatial social networks with "neighbourhoods" defined by Euclidian distance [14] and extending the variation in which particles move toward neighbouring particles that have found a good solution [1], a new algorithm was proposed, modelled on the behaviour of locusts and crickets. It adapted the ideas to multi-objective optimisation problems by making comparison between neighbouring particles based on Pareto dominance, and adding a corresponding repulsion to the previously suggested attraction. By confining influences on individual particles to Pareto-dominance interactions between nearest neighbours only, guide particles for the swarm were dispensed with altogether. It was demonstrated that this

Andrew Lewis is with the Institute for Integrated and Intelligent Systems, Griffith University, Queensland, Australia (email: a.lewis@griffith.edu.au). approach provided results comparable to a conventional multiobjective PSO (MOPSO) algorithm, but improved coverage of the Pareto-front. However, it was noted that Buhl et al. had identified a critical density for the transition from disordered to ordered behaviour in locust swarms [2] which suggested that this might be an important factor in models of behaviour of this type.

The remainder of this paper is organised as follows. Section II gives a brief summary of the proposed "LoCost" algorithm. The investigation of the effects of swarm size and, indirectly, population density on the new system is given in Section III and finally, the conclusions and future research directions are presented in Section IV.

\section{The LoCost Algorithm}

The movement of particles in conventional PSO algorithms is governed by two equations:

$$
\begin{gathered}
v_{i j}(t+1)=w v_{i j}(t)+c_{1} r_{1 j}(t)\left[y_{i j}(t)-x_{i j}(t)\right. \\
+c_{2} r_{2 j}(t)\left[\hat{y}_{i j}(t)-x_{i j}(t)\right. \\
x_{i j}(t+1)=x_{i j}(t)+v_{i j}(t+1)
\end{gathered}
$$

Here, $v_{i j}(t)$ is the velocity of particle $i$ in dimension $j$ at time $t$ and $x_{i j}(t)$ is the position of particle $i$ in dimension $j$ at time $t$. The velocity of a particle depends on both the best position that particle has found to time $t, y_{i j}(t)$, and the best solution the entire swarm has found has found to time $t, \hat{y}_{i j}(t)$.

PSO algorithms are based on observation of animal behaviour, with simple rules abstracted from this observation giving rise to sophisticated emergent behaviour. Simpson et al. [13] have observed cricket swarms and found that the marching crickets were nutrient deprived, specifically in protein and salt, and fed selectively to redress these deficiencies. The crickets themselves were "walking packages of protein and salt". So while crickets in the vanguard of the swarm might remedy their nutrient deficiencies by feeding from new habitat, the only chance others had was through cannibalism.

If we consider the population of particles in a MOPSO algorithm, the non-dominated particles will constitute a surface of the swarm, the Pareto-front. These may be considered the "vanguard" and, in fact, in conventional MOPSO algorithms motion of the swarm generally would be toward them. These particles can be considered "satiated" to some extent, having "fed" on newly explored regions. Others in the swarm can be considered to be still nutrient deprived, and will move toward their nearest (well-fed) neighbour, the closest source of nutrients. Braendler and Hendtlass have already proposed that 
TABLE I: Test functions

\begin{tabular}{|c|c|c|}
\hline Test & & Constraints \\
\hline ZDT1 & $\begin{array}{l}g\left(x_{2}, \cdots, x_{n}\right)=1+9\left(\sum_{i=2}^{n} x_{i}\right) /(n-1) \\
h\left(f_{1}, g\right)=1-\sqrt{f_{1} / g} \\
f_{1}\left(x_{1}\right)=x_{1} \\
f_{2}(\vec{x})=g\left(x_{2}, \cdots, x_{n}\right) \cdot h\left(f_{1}, g\right)\end{array}$ & $\begin{array}{l}x_{i} \in[0,1] \\
i=1,2, \ldots, n \\
n=2\end{array}$ \\
\hline ZDT2 & $\begin{array}{l}g\left(x_{2}, \cdots, x_{n}\right)=1+9\left(\sum_{i=2}^{n} x_{i}\right) /(n-1) \\
h\left(f_{1}, g\right)=1-\left(f_{1} / g\right)^{2} \\
f_{1}\left(x_{1}\right)=x_{1} \\
f_{2}(\vec{x})=g\left(x_{2}, \cdots, x_{n}\right) \cdot h\left(f_{1}, g\right)\end{array}$ & $\begin{array}{l}x_{i} \in[0,1] \\
i=1,2, \ldots, n \\
n=2\end{array}$ \\
\hline ZDT3 & $\begin{array}{l}g\left(x_{2}, \cdots, x_{n}\right)=1+9\left(\sum_{i=2}^{n} x_{i}\right) /(n-1) \\
h\left(f_{1}, g\right)=1-\sqrt{f_{1} / g}-\left(f_{1} / g\right) \sin \left(10 \pi f_{1}\right) \\
f_{1}\left(x_{1}\right)=x_{1} \\
f_{2}(\vec{x})=g\left(x_{2}, \cdots, x_{n}\right) \cdot h\left(f_{1}, g\right) \\
g\left(x_{2}, \cdots, x_{n}\right)=1+10(n-1)\end{array}$ & $\begin{array}{l}x_{i} \in[0,1] \\
i=1,2, \ldots, n \\
n=2\end{array}$ \\
\hline $2 D 14$ & $\begin{array}{l}\quad+\sum_{i=2}^{n}\left(x_{i}^{2}-10 \cos \left(4 \pi x_{i}\right)\right) \\
h\left(f_{1}, g\right)=1-\sqrt{f_{1} / g} \\
f_{1}\left(x_{1}\right)=x_{1} \\
f_{2}(\vec{x})=g\left(x_{2}, \cdots, x_{n}\right) \cdot h\left(f_{1}, g\right)\end{array}$ & $\begin{array}{l}x_{1} \in[0,1] \\
x_{i} \in[-5,5] \\
i=2, \ldots, n \\
n=2\end{array}$ \\
\hline
\end{tabular}

particles move toward neighbouring particles that have found good solutions [1]. But, of course, as Simpson et al. observed, these targets of cannibalism move away from their predatory conspecifics, if able. In terms of algorithm development this suggested that a motion vector away from a particle that has not found a good solution may be just as important as one toward good solutions. So the vector equation 1 of the conventional PSO was reformulated:

$v_{i j}(t+1)=\left\{\begin{array}{l}w v_{i j}(t)+c_{1} r_{1 j}\left(\overrightarrow{x_{i^{\prime}}}(t)-\overrightarrow{x_{i}}(t)\right): \overrightarrow{x_{i^{\prime}}}(t) \prec \overrightarrow{x_{i}}(t) \\ w v_{i j}(t)-c_{1} r_{1 j}\left(\overrightarrow{x_{i^{\prime}}}(t)-\overrightarrow{x_{i}}(t)\right): \overrightarrow{x_{i}}(t) \prec \overrightarrow{x_{i^{\prime}}}(t)\end{array}\right.$

i.e. motion is toward a neighbour if it is Pareto-dominant, and away otherwise. This interaction is between nearest neighbour pairs only, i.e. between the particle with index $i$ and its neighbouring particle, $i^{\prime}$, where the Euclidian distance between them, $\mathcal{E}\left(\overrightarrow{x_{i}}, \overrightarrow{x_{i^{\prime}}}\right)$, is a minimum.

This form of the algorithm has been tested on a number of well known test functions taken from Zitzler et al. [15]. It was found that the proposed algorithm, LoCost, performed quite comparably to a conventional MOPSO algorithm in terms of convergence, and achieved appreciably greater coverage of the approximation to the Pareto-front than the MOPSO algorithm, "filling the gaps" in a number of places [9].

\section{Computational Experiments And Results}

As noted, Buhl et al. identified a critical density for the transition from disordered to ordered behaviour in locust swarms [2]. To investigate a potential dependence on swarm size, and the resulting population density implied, the LoCost algorithm was compared to a conventional MOPSO algorithm, with Sigma update of guide particles [10], for several different population sizes. Separate runs, with different random seeds, were performed on standard test functions. The test functions used have a variety of convex, non-convex, discontinuous and multimodal Pareto-fronts and are shown in Table I. The dimension of parameter space, $N$, was 2 for all test functions.

To illustrate the results achieved, Figures 1 to 3 show the approximations to the Pareto-front achieved by the archive members of the LoCost and MOPSO algorithms after 80 generations for the convex, ZDT1 test function with a swarm size of 25,50 , and 100 , respectively.

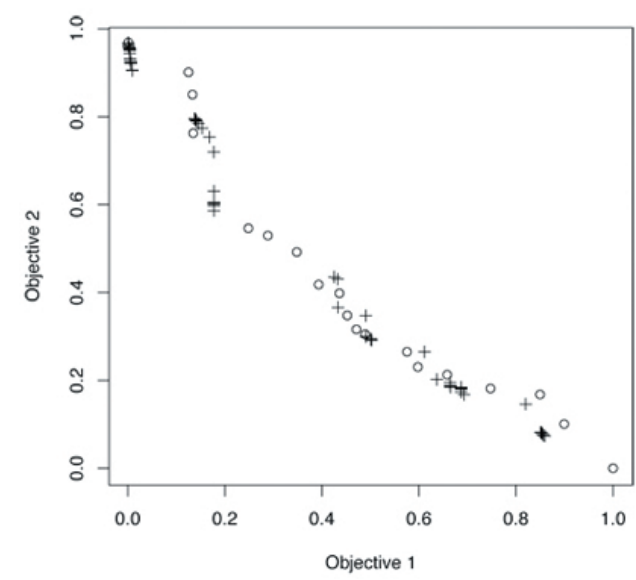

Fig. 1: Approximate Pareto-front for the ZDT1 test function at 80 iterations for a swarm size of 25. (MOPSO shown as "+", LoCost as “o”)

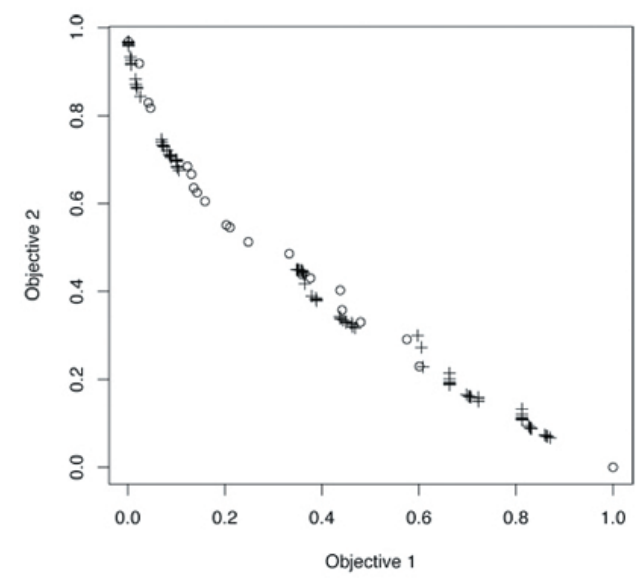

Fig. 2: Approximate Pareto-front for ZDT1 test function at 80 iterations for a swarm size of 50. (MOPSO shown as “+”, LoCost as "o")

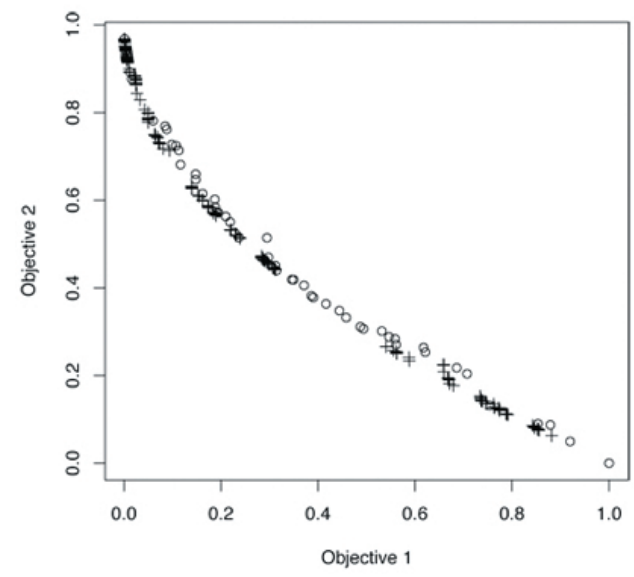

Fig. 3: Approximate Pareto-front for the ZDT1 test function at 80 iterations for a swarm size of 100. (MOPSO shown as "+", LoCost as "o") 
By inspection of the figures it may be seen that the approximation to the Pareto-front of the attainment surface for both algorithms improves markedly with increasing swarm size. Figure 1 shows both algorithms to be patchy in their coverage and only partly converged over much of the front for a swarm size of 25. By the time the swarm size is 100 , both algorithms demonstrate a more fully-converged attainment surface, closely approximating the convex shape of the Pareto-front. Comparison of the attainment surfaces of both algorithms for the non-convex and discontinuous test functions shows a similar trend.

It might be argued that such a result is unremarkable, since at 80 iterations a swarm of 100 particles will have sampled and evaluated 4 times as many points in the search space as a swarm of only 25 particles. To test whether it was just the total number of test solutions evaluated that was producing the qualitative differences between different runs of the algorithms with different swarm sizes, the experiment was repeated, with the swarm of 25 allowed 80 iterations, the swarm of 50 allowed 40 iterations, and the swarm of 100 allowed only 20 iterations, i.e. each swarm allowed an equivalent number of evaluations. The results are compared in Figures 4 to 6 . Once more, the attainment surfaces achieved by algorithms with larger swarm sizes appear closer approximations to the Pareto-front. Figures 7 to 9,10 to 12 and 13 to 15 show the corresponding results for the ZDT2, ZDT3 and ZDT4 test functions, respectively. The improved convergence with population size is particularly apparent with the multimodal, ZDT4 test function.

From the figures it would appear there is little to choose between the two algorithms in terms of convergence; both appear to approach the Pareto-front equally well at equivalent stages. The significant difference between the algorithms appears to be the degree of coverage achieved. A qualitative comparison appears to show that LoCost has an appreciably greater coverage of the approximation to the Pareto-front than the MOPSO algorithm, "filling the gaps" in a number of places and extending the range of objective function values the attainment surface covers. To quantitatively evaluate the coverage of each method a performance measure, $\Psi$, introduced in an earlier paper [9] was again employed.

Briefly, for a two-dimensional problem, one of the objectives is selected and the respective dimension of the objective space is split into a number of equally sized intervals, $\psi_{n}$. Each of these intervals is inspected to see if a non-dominated solution lies between the upper and lower bounds of the interval. If at least one such particle exists, the interval is scored 1 , otherwise its score is set to 0 . The metric is given by the percentage of "occupied" intervals. This metric is a measure of the total extent of the Pareto-front covered, and the uniformity of the coverage. It may be applied where the true Pareto-front is not known, the coverage being measured relative to the total, aggregated attainment surfaces of the two algorithms being compared. In this respect it is more generally applicable than the Spread metric defined by Deb et al. [3] and is quite simple to implement.

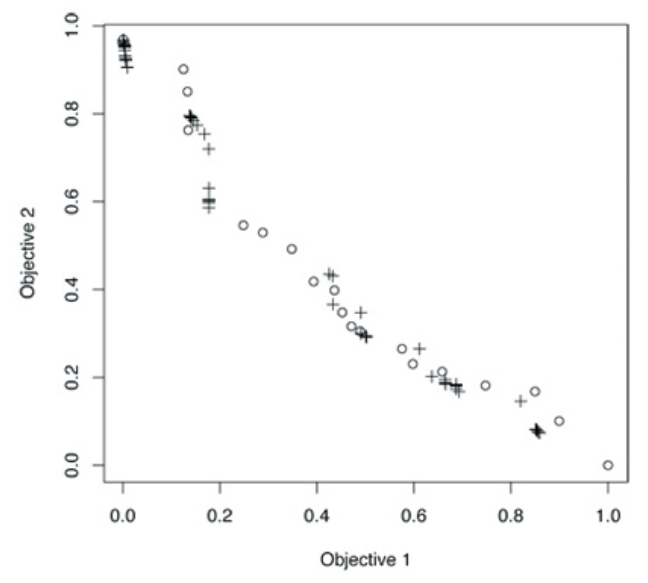

Fig. 4: Approximate Pareto-front for the ZDT1 test functions at 80 iterations for a swarm size of 25. (MOPSO shown as "+", LoCost as "o")

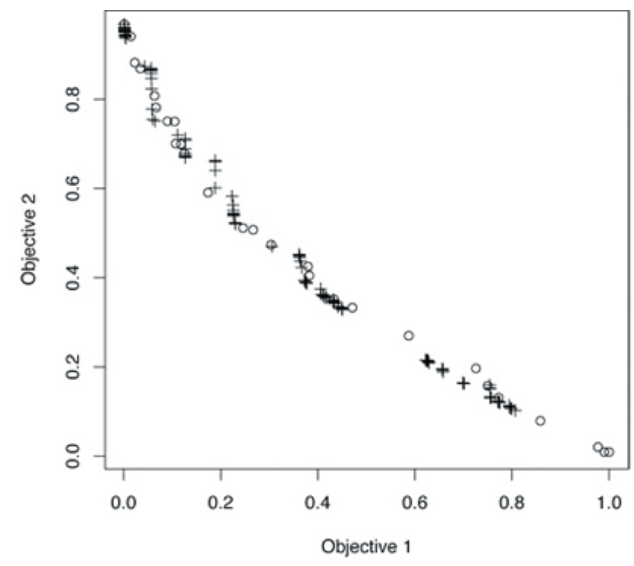

Fig. 5: Approximate Pareto-front for the ZDT1 test function at 40 iterations for a swarm size of 50. (MOPSO shown as "+", LoCost as "o")

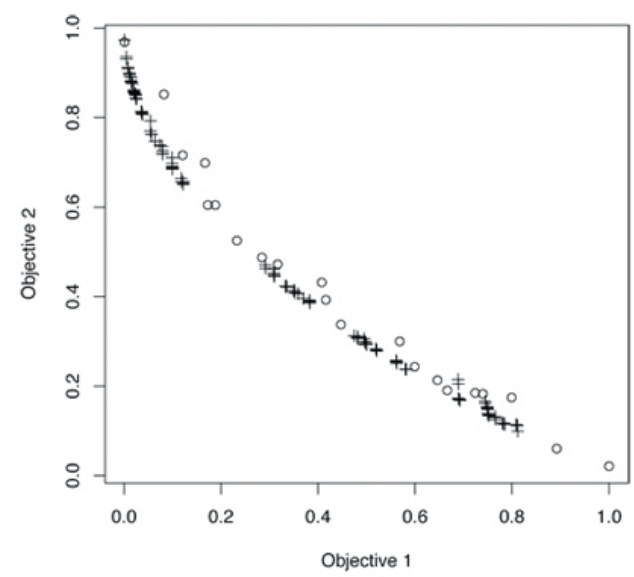

Fig. 6: Approximate Pareto-front for the ZDT1 test function at 20 iterations for a swarm size of 100. (MOPSO shown as "+", LoCost as "o") 


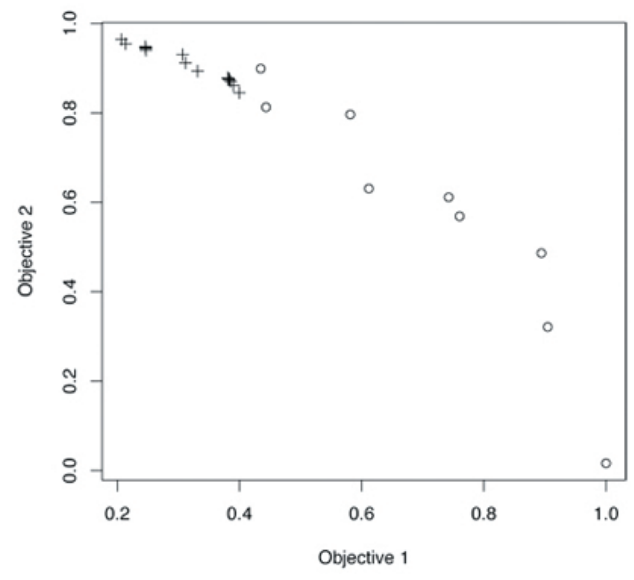

Fig. 7: Approximate Pareto-front for the ZDT2 test function at 80 iterations for a swarm size of 25. (MOPSO shown as “+”, LoCost as "o")

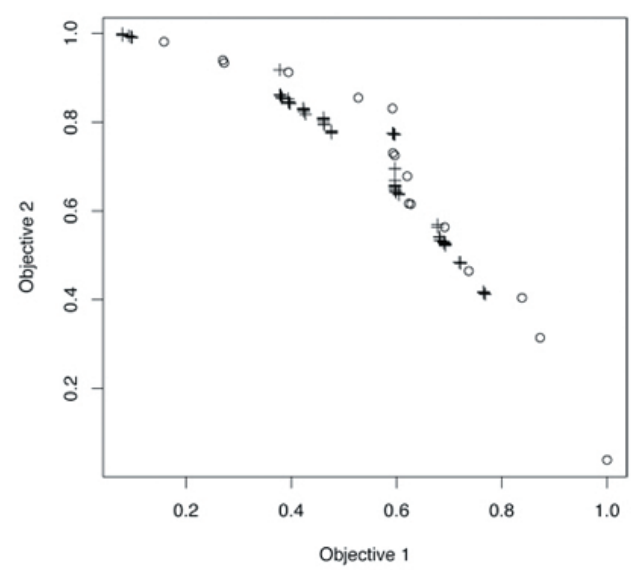

Fig. 8: Approximate Pareto-front for the ZDT2 test function at 40 iterations for a swarm size of 50. (MOPSO shown as “+”, LoCost as “o")

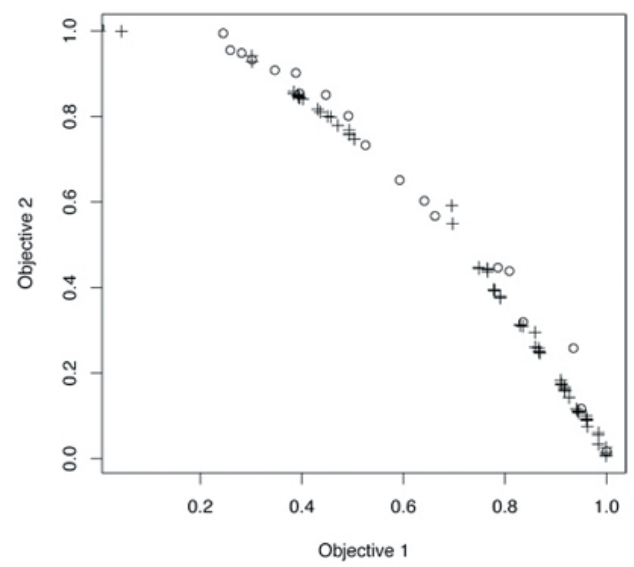

Fig. 9: Approximate Pareto-front for the ZDT2 test function at 20 iterations for a swarm size of 100. (MOPSO shown as "+", LoCost as "o")

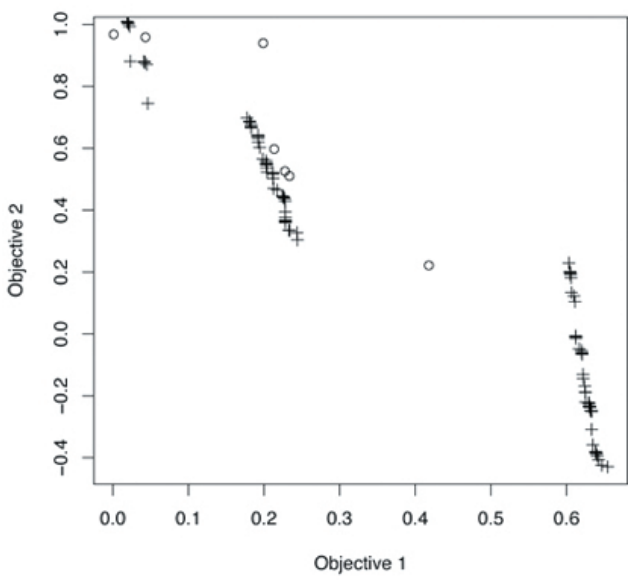

Fig. 10: Approximate Pareto-front for the ZDT3 test functions at 80 iterations for a swarm size of 25. (MOPSO shown as “+”, LoCost as "o")

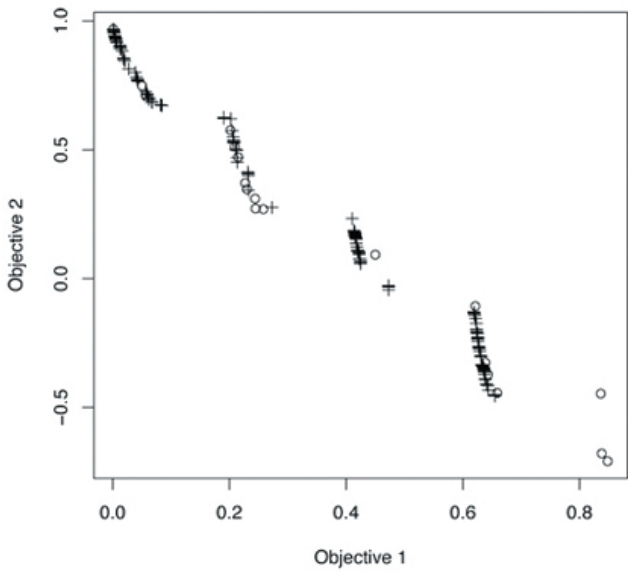

Fig. 11: Approximate Pareto-front for the ZDT3 test function at 40 iterations for a swarm size of 50. (MOPSO shown as "+", LoCost as "o")

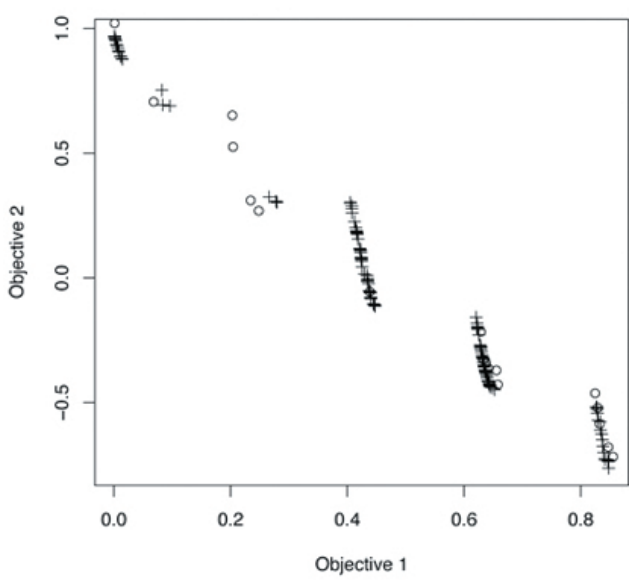

Fig. 12: Approximate Pareto-front for the ZDT3 test function at 20 iterations for a swarm size of 100. (MOPSO shown as "+", LoCost as "o") 


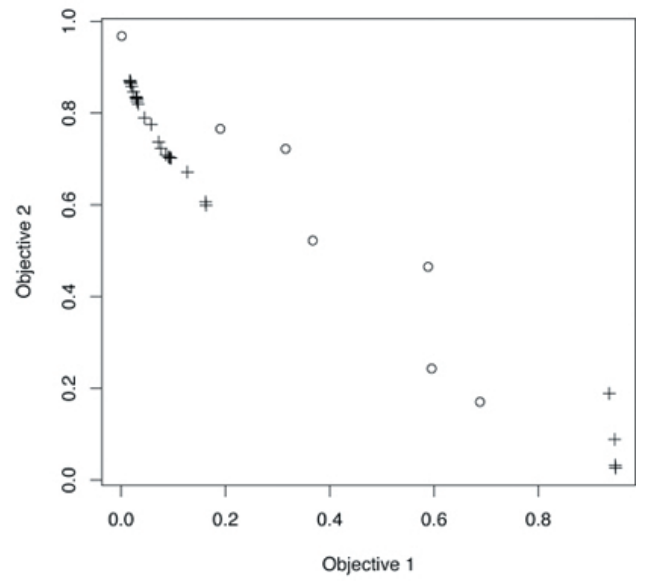

Fig. 13: Approximate Pareto-front for the ZDT4 test functions at 80 iterations for a swarm size of 25. (MOPSO shown as “+”, LoCost as "o")

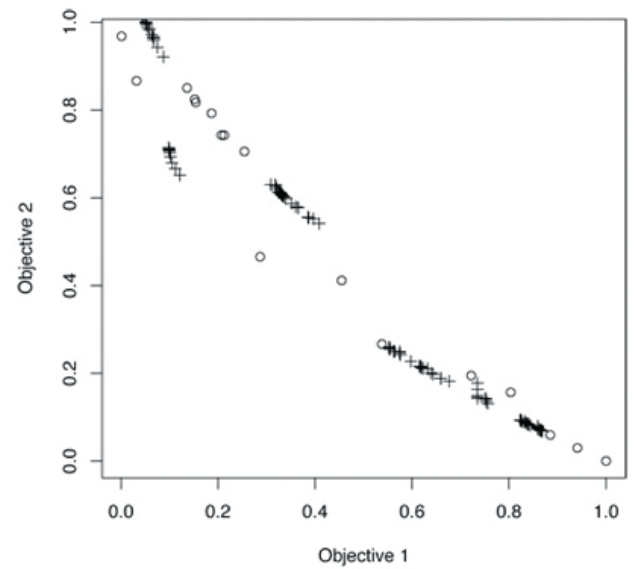

Fig. 14: Approximate Pareto-front for the ZDT4 test function at 40 iterations for a swarm size of 50. (MOPSO shown as "+", LoCost as "o")

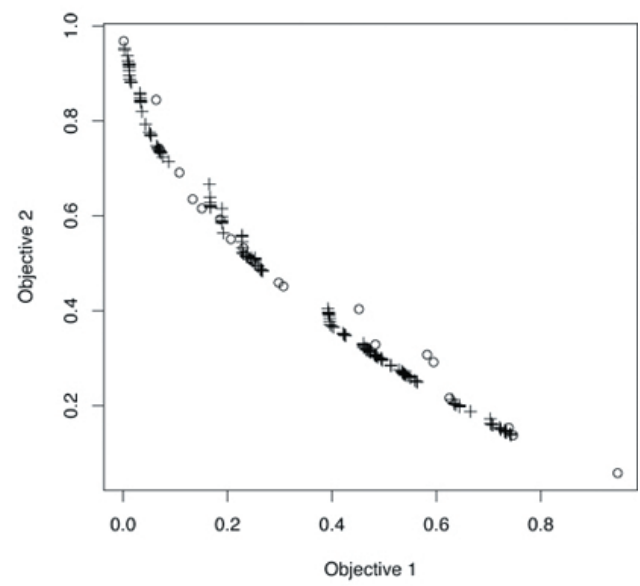

Fig. 15: Approximate Pareto-front for the ZDT4 test function at 20 iterations for a swarm size of 100. (MOPSO shown as “+”, LoCost as "o")
In the figures it may be seen that the MOPSO algorithm has a tendency to accumulate many, densely packed points on the attainment surface, particularly for larger populations. In contrast, LoCost has fewer, more evenly scattered points. To avoid biasing results, an interval equivalent to $5 \%$ of the total extent of the attainment surface was used. Values of the coverage metric for each algorithm on each test function, at 80,40 and 20 iterations for populations of 25,50 and 100 , respectively, are displayed in Table II.

TABLE II: Coverage Metric, $\Psi$, for equal numbers of evaluations

\begin{tabular}{ccccccccc}
\hline $\begin{array}{l}\text { Swarm } \\
\text { Size }\end{array}$ & \multicolumn{4}{c}{ LoCost } & \multicolumn{5}{c}{ MOPSO } \\
\hline & ZDT1 & ZDT2 & ZDT3 & ZDT4 & ZDT1 & ZDT2 & ZDT3 & ZDT4 \\
\hline 25 & 60 & 25 & 25 & 24 & 50 & 25 & 35 & 24 \\
50 & 75 & 45 & 40 & 30 & 65 & 35 & 45 & 50 \\
100 & 75 & 60 & 35 & 19 & 65 & 55 & 45 & 38 \\
\hline
\end{tabular}

By reference to Table II it may be seen that the LoCost algorithm does, indeed, exhibit better coverage than the MOPSO algorithm for all swarm sizes on ZDT1 and ZDT2. MOPSO has better measured coverage for ZDT3, despite almost completely missing one of the Pareto-front segments in each experiment. LoCost more reliably detected each segment, but in some cases failed to adequately cover the extent of the Pareto-front. Both algorithms show increased "coverage" for ZDT4 with a swarm size of 50, but inspection of Figure 14 shows this was at the expense of convergence. Despite these caveats, there is a clear trend for both algorithms toward improved coverage for larger swarm size (and, by implication, increased population density, or crowding.) On the evidence to hand there appears to be some critical transition between population sizes of 25 and 50, where there is generally a marked increase in coverage attained, compared to the transition from 50 to 100 where, in several cases, the coverage plateaued or even, in the case of ZDT4, reduced.

As the performance of both algorithms appeared to be affected by population density, and suffering poor convergence at lower population sizes, it may be instructive to consider a quantitative measure of convergence. A simple measure is to consider the average distance of the attainment surface to a utopia point. These results, for each of the test cases and population sizes, and using equal numbers of evaluations in each case, are shown in Table III. As with the coverage metric, a general trend toward better performance with greater population size may be seen. With only a couple of exceptions, there is a marked improvement as a result of increasing the population from 25 to 50 , and a tapering off the rate of increase as population size is increased further.

The beneficial effects of crowding on the performance of both algorithms may have significant implications for algorithm design in distributed computing environments. Particularly for the Island model, greater numbers of participating nodes can lead to significantly smaller, individual swarms since the goal of distributing the computation is to reduce the 
TABLE III: Convergence for equal numbers of evaluations, as average distance to Utopia point. (Smaller value is better.)

\begin{tabular}{ccccccccc}
\hline $\begin{array}{l}\text { Swarm } \\
\text { Size }\end{array}$ & \multicolumn{3}{c}{ LoCost } & \multicolumn{5}{c}{ MOPSO } \\
\hline & ZDT1 & ZDT2 & ZDT3 & ZDT4 & ZDT1 & ZDT2 & ZDT3 & ZDT4 \\
\hline 25 & 0.72 & 0.97 & 0.77 & 0.96 & 0.81 & 0.98 & 0.66 & 1.09 \\
50 & 0.75 & 0.95 & 0.66 & 0.80 & 0.72 & 0.92 & 0.66 & 0.88 \\
100 & 0.70 & 0.95 & 0.76 & 0.67 & 0.71 & 0.93 & 0.68 & 0.72 \\
\hline
\end{tabular}

elapsed time for the optimisation by concurrently executing a smaller number of fitness evaluations on each of several nodes. From the results presented here it may be concluded that this speedup of the computation could come at the cost of correspondingly degraded algorithmic performance. This suggests that Master-Slave models may be better suited for distributed PSO, though care should be taken to ensure fault tolerance of the algorithms in unreliable, distributed environments [12].

\section{CONClusions}

The experiments described in this paper have demonstrated that swarm size and, implicitly, population density, significantly impact the performance of both a new, spatial, social network optimisation algorithm inspired by the swarming behaviour of crickets and locusts, and a conventional MOPSO algorithm. This suggests that care should be taken in the design of distributed particle swarm optimisation algorithms lest reduced individual swarm sizes negatively impact algorithm performance.

The results reported in this paper are preliminary. The coverage and convergence achieved by both algorithms improved with population size. The statistical significance of the features shown should be confirmed, but the results observed would appear to warrant further investigation.

Fernandes et al. [5] have found artificial foraging swarms with freely varying population sizes may reach self-regulated equilibria. The specific relationship between swarm size and actual population density in localised regions, and the effect on the search efficacy of the LoCost algorithm, is of particular interest. Future work will include further investigation of the behaviour of LoCost and its dependence on several factors.

\section{REFERENCES}

[1] D. Braendler and T. Hendtlass. The suitability of particle swarm optimization for training neural hardware. In Proc. 15th International Conference on Industrial and Engineering Applications of Artificial Intelligence and Expert Systems, volume 2358 of LNCS, pages 190199. Springer-Verlag, 2002.

[2] J. Buhl, D. Sumpter, I. Couzin, J. Hale, E. Despland, E. Miller, and S. Simpson. From disorder to order in marching locusts. Science, 312:1402-1406, 2006.

[3] K. Deb., A. Pratap, S. Agarwal, and T. Meyarivan. A fast and elitist multiobjective genetic algorithm: NSGA-II. Evolutionary Computation, IEEE Transactions on, 6(2):182-197, 2002.

[4] A. Engelbrecht. Fundamentals of Computational Swarm Intelligence. Wiley, 2005.

[5] C. Fernandes, V. Ramos, and A. Rosa. Varying the population size of artificial foraging swarms on time varying landscapes. In Artificial Neural Networks: Biological Inspirations - ICANN 2005, volume 3696/2005 of LNCS. Springer-Verlag, 2005.

[6] S. Guru, S. Halgamuge, and S. Fernado. Particle swarm optimisers for cluster formation in wireless sensor networks. In Proc. 2005 International Conference on Intelligent Sensors, Sensor Networks and Information Processing Conference, pages 319-324, 2005.

[7] D. Ireland, A. Lewis, S. Mostaghim, and J. Lu. Hybrid particle guide selection methods in multi-objective particle swarm optimization. In Proc. 2nd IEEE International Conference on eScience and Grid Computing, 2006.

[8] J. Kennedy and R. Eberhart. Particle swarm optimization. In Proc. International Conference on Neural Networks, 1995.

[9] A. Lewis. LoCost: a spatial social network algorithm for multi-objective optimisation. In Proc. IEEE Congress on Evolutionary Computation (CEC 2009), 2009.

[10] S. Mostaghim and J. Teich. Strategies for finding good local guides in multi-objective particle swarm optimization (MOPSO). In Proc. IEEE Conference on Swarm Intelligence Symposium, pages 26-33, 2003.

[11] Y. Rahmat-Sammi, N. Jin, and S. Xu. Particle swarm optimization (pso) in electromagnetics: Let the bees design your antennas. In 22nd Annual Review on Progress in Applied Electromagnetics, 2006.

[12] I. Scriven, D. Ireland, A. Lewis, S. Mostaghim, and J. Branke. Asynchronous multiple objective particle swarm optimisation in unreliable distributed environments. In Proceedings of the IEEE Congress on Evolutionary Computation (CEC 2008), 2008.

[13] S. Simpson, G. Sword, P. Lorch, and I. Couzin. Cannibal crickets on a forced march for protein and salt. Proc. of the National Academy of Sciences of the United States of America (PNAS), 103(11):4152-4156, 2006.

[14] P. Suganthan. Particle swarm optimiser with neighborhood operator. In Proc. IEEE Congress on Evolutionary Computation, pages 1958-1962, 1999.

[15] E. Zitzler, K. Deb, and L. Thiele. Comparison of multiobjective evolutionary algorithms on test functions of different difficulty. In Proc. 1999 Genetic and Evolutionary Computation Conference. Workshop Program, pages 121-122, 1999. 